\title{
Geometrical characterization of various shaped 3D-aggregates of primary spherical particules by radial distribution functions
}

\author{
Marthe Lagarrigue ${ }^{1}$, Johan Debayle ${ }^{2}$, Sandra Jacquier ${ }^{1}$, Frédéric Gruy ${ }^{1}$, and \\ Jean-Charles Pinoli ${ }^{2}$ \\ 1 SPIN/LPMG, UMR CNRS 5148 \\ ${ }^{2}$ CIS/LPMG, UMR CNRS 5148 \\ Ecole Nationale Supérieure des Mines de Saint-Etienne, \\ 158 cours Fauriel, 42023 Saint-Etienne cedex 2, France.
}

\begin{abstract}
Multi-scale aggregates are composed of particles which results themselves of agglomeration of other primary particles. If particles are modeled by their centers, the geometrical characterization of aggregates refers to point pattern analysis. Radial distribution and function of pairs allow a description of the point pattern to be performed. They describe how points are radially packed around each other. In this paper, the characterization of different simulated aggregates are computed and compared.
\end{abstract}

\section{Introduction}

In precipitation process, the final product is often obtained in the form of aggregates of particles, which themselves consist of assembling of smaller crystals. The purpose of this research work is to characterize 3D solid aggregates by a morphological method. Ultimately, this characterization will be related with an optical method which consists in analysing the scattering parameter of an aggregate under an incident light beam. Indeed, the scattering parameter particularly depends on the internal and external geometry of the aggregates e.g. the chord length distribution (see, for instance Jacquier and Gruy [1]). The final aim of this study is to find a link between the underlying optical and morphological parameters.

This paper is focused on the geometrical characterization of aggregates. Two methods of morphological characterization of the internal and external geometry are proposed: the radial distribution function, and the function of pairs.

Several experimental studies are then performed with computationally simulated aggregates. A comparison of the two proposed methods is carried out for aggregates constituted by different shape of convex hulls, different ratios of filling, and different geometrical shape ratios. 


\section{$2 \quad 3 \mathrm{D}$ aggregates modelling}

To study the aggregates morphology, it is necessary to simulate them in order to understand the influence of several geometrical parameters.

First of all, an aggregate is defined by its scale number. In this paper only the case of aggregates with two scales is presented.

1. The smaller scale level consists in spherical particles (imposed by the optical model [2]). The centers of these primary particles are distributed along the close-packed hexagonal mesh [4], selected for its compactness. The radius is chosen equal to $10 \mathrm{~nm}$ because it is the usual order of magnitude for primary particles of the first scale level in the optical domain.

2. The second scale level is defined by geometrical shapes: sphere, cube, cylinder, spheroïds (oblate and prolate). The cylindrical convex hull is defined by its base diameter and its height which is k-proportional to the base diameter, with $k \in\{1 ; 2 ; 8 ; 20\}$.

The geometry of the two spheroïdal convex hulls (oblate and prolate) are defined by the axis a, b and $\mathrm{c}$, with an equality between two axis lengths $(a=b$ for example). The third parameter, c, is proportional to the first one by a factor $\mathrm{k}, k>1$ for the prolate and $0<k<1$ for the oblate, respectively. In this paper will be used $k \in\{2 ; 20\}$ for the prolate case and $k \in\{1 / 2 ; 1 / 20\}$ for the oblate case.

Some examples of aggregates are shown in the figure 1.

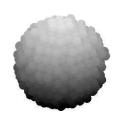

(a)

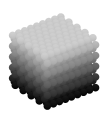

(b)

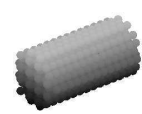

(c)

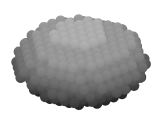

(d)

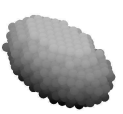

(e)

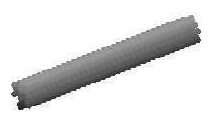

(f)

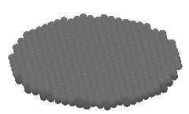

(g)

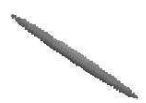

(h)

Fig. 1. Representation of different aggregates with a ful convex hull: (a) spherical, (b) cubic, (c) cylindrical with $k=2$, (d) oblate with $k=1 / 2$, (e) prolate with $k=2$, (f) cylindrical with $k=20,(\mathrm{~g})$ oblate with $k=1 / 20$, (h) prolate with $k=20$

Moreover, in order to compare the aggregates, the volume of their convex hull is the same value for all of them. This volume is fixed equal to that of a sphere with a $300 \mathrm{~nm}$ diameter, because this size is an usual order of magnitude 
of the second scale length in the optical domain.

The last studied parameter is the filling ratio of the convex hull by spherical primary particles: $100 \%, 75 \%, 50 \%$ and hollow aggregates.

The $100 \%$ filling aggregate is composed of particles whose center is inside the convex hull. This convex hull is placed so that it would be as fulfilled as possible. The method is shown in the figure 2).

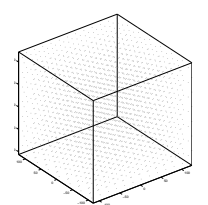

(a) Mesh

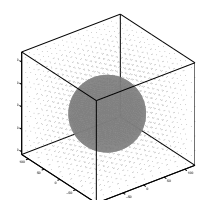

(b) Con-

vex hull

positioning

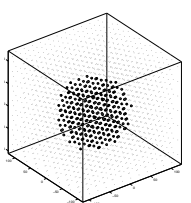

(c) Particle centers selection

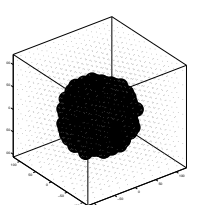

(d) Selected particles

Fig. 2. Building of the $100 \%$ filling spherical convex hull

The $75 \%$ et $50 \%$ random filling correspond to a random choice (standard uniform law) of, respectively, $75 \%$ and $50 \%$ of the particles selected in the $100 \%$ filling case.

Concerning the full aggregate, each primary particles has 12 adjacent primary particles, implied by the closed-packed hexagonal mesh, except the ones located on the aggregate's surface. So, the particles constituting the hollow aggregates are those of the corresponding aggregates with a full convex hull, which doesn't have their 12 neighbours.

The figure 3 illustrate the different filling ratios for the spherical convex hull.

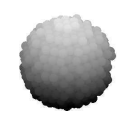

(a)

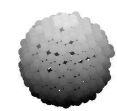

(b)

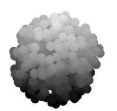

(c)

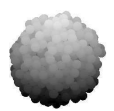

(d)

Fig. 3. Representation of aggregates with a spherical convex hull and different filling ratio: (a) full filling, (b) hollow filling, (c) random filling at 50\%, (d) random filling at $75 \%$

After aggregates simulation, the study of their geometrical characterization using two methods is performed in the next section. The particles are modeled by their center. As a consequence the aggregate is analysed such as a distribution of points (point pattern analysis). 


\section{Geometrical characterization}

Firstly, for each method, some results are presented to compare the different filling ratios (explained below): this is done for only one type of convex hull (the spherical convex hull) because the comments done for one are similar for the other ones. Next, analogies and differences between aggregates with quasisimilar convex hull are analyzed: spherical, cubic, cylindrical with $k=2$, oblate with $k=1 / 2$ and prolate with $k=2$. Lastly, the cylindrical convex hull with several $k$-parameter values are compared.

\subsection{Radial distribution (RD)}

The radial distribution (RD) method uses a sphere $S$, the center of which is chosen within the aggregate, and the radius $r$ of which is variable. The value of $r$ starts from 0 and then increases until the sphere totally incircles the aggregate. For each $r$ value, the number of particle centers included in $S$ is calculated. The same process could be done with the particle volume (quantity of matter), included in $S$ as shown in Fig.4 with an aggregate constituted of non-connected particles. The study is focused on the distribution of the particle centers. Therefore, the cumulative radial distribution function (CRDF) can be extracted with regard to the parameter $r$. In this paper, the center of $S$ is the geometrical center of the aggregate. Concerning the discretization of the $r$ value, the step between two $r$ values is fixed to $20 \mathrm{~nm}$, because it is the smallest distance between two particle centers, the radius of one particle being equal to $10 \mathrm{~nm}$.

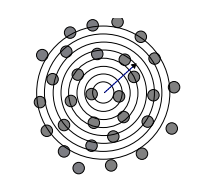

Fig. 4. Process of radial distribution function with an increasing radius $\mathrm{r}$

Mathematically, the formula for the CRDF is defined by:

$$
C R D F(r)=\frac{\text { Number of particle centers at a distance } \leq r}{\text { Total number of particles in the aggregate }}
$$

Since the aggregates have similar volume and are built along the same mesh, the focus has been placed on the particle mean number, normalized or not by the total number of particle within the aggregate.

\section{Characterization of the filling ratios of one convex hull}

Fig.5 shows the CRDF for the spherical convex hull aggregates with different filling ratios. 


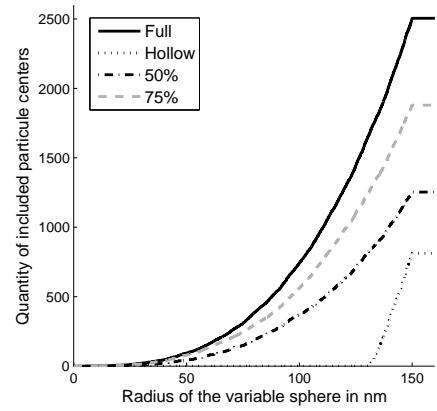

(a) Non-normalized results

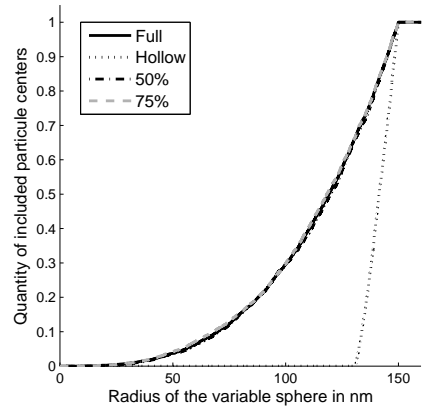

(b) Normalized results

Fig. 5. Radial cumulative distribution function for the aggregates with a spherical convex hull and different filling ratios. The graph (a) is non-normalized. In (b) the number of centers included in $S$ is normalized $(\mathrm{CRDF}(\mathrm{r}))$ by the total number of particles within each aggregate.

In Fig.5(a), it can be noticed that random filled aggregates are uniformely filled, because for each value of $r$, the ratios $50 \%$ and $75 \%$ are conserved between the concerned curves, until the r-value equal to the convex hull radius.

In Fig.5(b), for aggregates with full convex hull, or filled at $75 \%$ or at $50 \%$ respectively, the normalized number of inclusion follows the same curve. This curve's equation is $f(r)=\frac{r^{3}}{R^{3}}$, where $r$ and $R$ are the radius of the sphere $\mathrm{S}$, and the radius of the spherical hull, respectively. This equation comes from the fact that the distribution of the centers is uniform (standard uniform law). Consequently, the normalized radial cumulative distribution function with the aggregate is calculated as the volume of the sphere $\mathrm{S}$, normalized by the volume of the convex hull of the aggregate.

However, this is different for the aggregate with a hollow convex hull. Indeed, the particles are along the convex hull so that the centers are included at the same step. It is the reason why, the curve for the hollow spherical convex hull is (theorically) a Heaviside function (see Fig. 5(b)).

\section{Characterization of aggregates of quasi-similar convex hulls}

Spherical, cubic, cylindrical, prolate with $k=2$ and oblate $k=1 / 2$ (Fig.7) convex hull aggregates are now studied. 


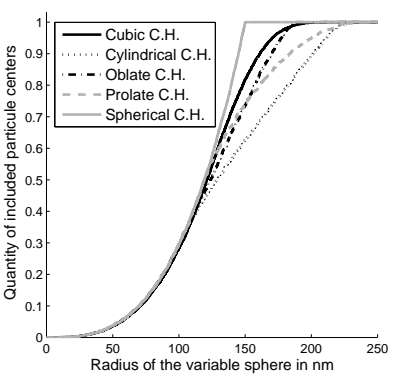

(a)

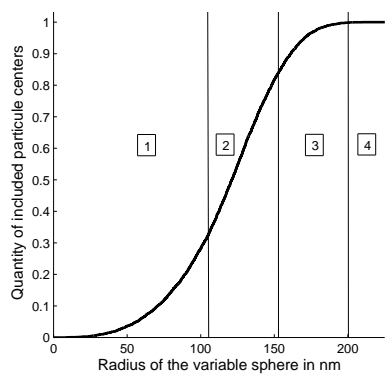

(b)

Fig. 6. (a) CRDF for quasi-similar and full convex hull aggregates. (b) CRDF for only the cubic convex hull.

The CRDF curves corresponding to each type of convex hull are different. All the curves have a common part: there, $S$ is included within the aggregate. The equation of the radial cumulative distribution function is linked to the volumic fraction of the variable sphere $S$ (Fig.5(b)). It is the reason why four phases in the curve corresponding to the cubic convex hull aggregate can be observed as in Fig.6(b) and Fig.7.

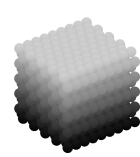

(a)

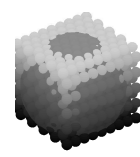

(b)

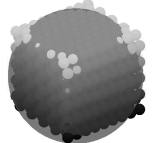

(c)

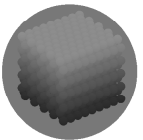

(d)

Fig. 7. For the cubic convex hull, visualisation of the different phases CRDF curve

The first graph (Fig. 7(a)), is identical for all the type of convex hull: this is the phase where the variable sphere is totally included in the aggregate.

The second phase (Fig. 7(b)), is when the sphere overflows the aggregate forming spherical caps. The form of the cap basis depends on the aggregate convex hull: it is plane for the cubic convex hull, but, for example, curved for the oblate. In some cases, there may be a third phase (Fig. 7(c)), where the caps begin to join, even if the aggregate is not totally incircled. For example, concerning the cubic convex hull, caps join before that the corners would be inside the variable sphere S. The ultimate phase (Fig. $7(\mathrm{~d})$ ) starts when the aggregate is totally incircled.

Characterization of aggregates with the same convex hull and several aspect ratios 
The figure 8 shows the results for a cylindrical convex hull with several values for the parameter $k$ (shape ratio).

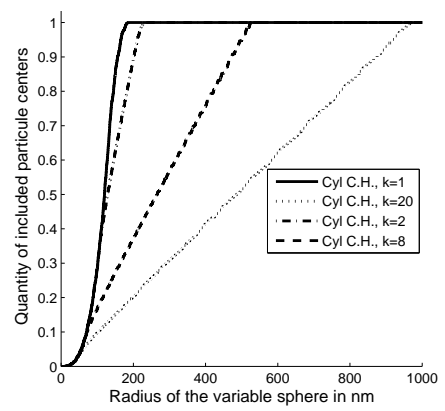

Fig. 8. CRDF for cylindrical and full convex hull aggregates with several shape ratio $k$

As mentioned for the previous graph, four phases of the cumulative radial distribution function curve can be seen (Fig.9), especially for the case with $k=1$. These four stages can be also observed for $k \neq 1$ cases. As all the convex hulls have the same volume, the larger $k$ is, the shorter the base diameter is (and longer the heigth is). Thus the more large is $k$, the longer the second phase is, contrary to the others phases.

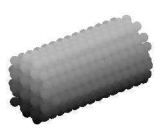

(a)

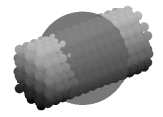

(b)

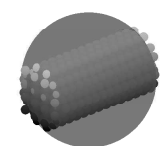

(c)

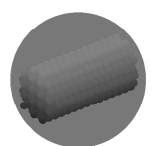

(d)

Fig. 9. Four phases for CRDF of the cylindrical convex hull aggregates

To conclude on this first quantification method, the radial distribution method allows to differenciate the external structure of an aggregate.

\subsection{Function of pairs}

The functions of pairs are morphological functions developped by means of integral geometry in Santalo [5]. They act as radial distribution functions, but are applied to each center of the particles constituting an aggregate. A pair designates the distance between a couple of particle centers. This function is closed to Ripley's function exposed in [6]. In the works of Gruy [7] are expressed the 
analytical pairs distribution functions of a spheroïd, oblate and prolate. In this paper, a simulated cumulative distribution of inter-center distances (averaged over the total number of pairs) is then computed.

The mathematical formula of the cumulative pair distribution function (CPDF) is:

$$
C P D F(r)=\frac{N u m b e r \text { of pairs } \leq r}{\text { Total number of pairs in the aggregate }}
$$

\section{Characterization of the filling ratios of one convex hull}

The results for the spherical convex hull aggregates ar shown in Fig.10.

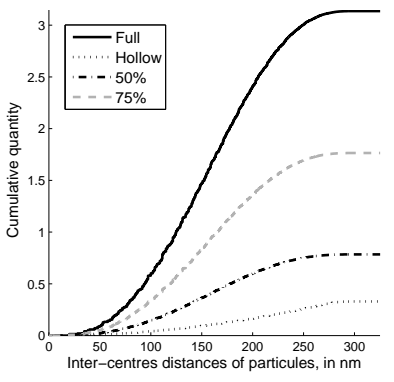

(a) Non-normalized results

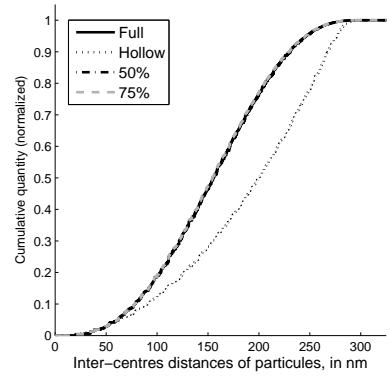

(b) normalized results

Fig. 10. Inter-center distances distribution for the aggregates of spherical convex hull, with different filling ratios. (a) non-normalized case. (b) Distribution normalized by the total number of inter-centers distances of each aggregate respectively, i.e. CPDF

As in the CRDF, the CPDF does not distinguish the filling ratio. Indeed, after normalization by the total number of inter-center distances of each aggregate respectively, the curves of $100 \%, 75 \%$ and $50 \%$ exactly coincide. Besides, the curve of the hollow convex hull aggregate remains isolated.

In Fig.10, especially in Fig.10(b), a inflection point of the curves can be noticed. It means that, for the spherical convex hull aggregate, there is a particular inter-center distance (about $150 \mathrm{~nm}$ ), which is the same for full, $75 \%$ and $50 \%$ filling ratios, and another particular inter-center distance for the hollow spherical convex hull which is equal to $250 \mathrm{~nm}$.

\section{Characterization of aggregates of quasi-similar convex hulls}

The results obtained for quasi-similar full convex hull aggregates are compared (Fig.11).

The CPDF for pherical, cubic, cylindrical, prolate with $k=2$ and oblate with $k=1 / 2$ convex hull aggregates are calculate and shown in the figure 11 . 


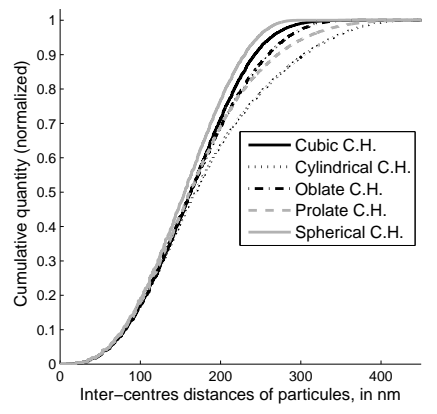

Fig. 11. CPDF for quasi-similar convex hull and full aggregates (spherical, cubic, cylindric with $\mathrm{k}=2$, oblate with $\mathrm{k}=1 / 2$, prolate with $\mathrm{k}=2=$ )

As in the figure 10, an inflection point is also observed at some inter-center distance values in the figure 11 . These values are the same for the different convex hulls presented, and corresponds to a statistical mode (a class of the distribution having the maximum of elements).

\section{Characterization of aggregates with the same convex hull type and} several shape ratios

A similar inflection point can be remarked in the figure 12, which represents the results for aggregates with a cylindrical of convex hull, and different values for the $k$-parameter $(1,2,8,20)$.

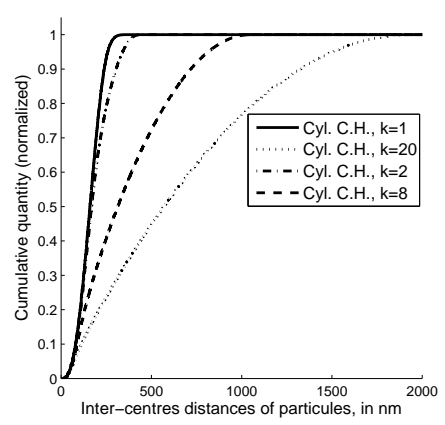

Fig. 12. CPDF for full and cylindrical convex hull aggregates, with different shape ratio $k$

Firstly, a proportionality between the largest inter-center distance values of each cylindrical convex hull aggregate and the $k$-parameter can be confirmed by the figure 12 . 
Further, the inflection point can be located for smaller inter-distance value while $\mathrm{k}$-parameter increases.

The inter-center distance corresponding to the inflection point, and the maximal inter-center distances of each CPDF characterize the isotropy of the aggregate shape. These relation between these two elements characterize if the aggregate is hunched up (cubic, oblate convex hulls) or if the hull presents extensions (cylindric convex hull with $\mathrm{k}=8$ or $20 \ldots$ ), or anisotropies.

\section{Conclusion and perspectives}

This article deals with two statistical methods for the morphological characterization of an aggregate of spherical particles. In a first time, cumulative radial distribution function allows an external analysis of the convex hull aggregate to be performed. In addition, this function is linked with the volumic fraction of the sphere S, normalized by the volume of the convex hull of the aggregate. It would be interesting to find analytically the equation of this function. On a second time, the analysis of the cumulative distribution function of pairs, i.e. distances between all of the particle centers of an aggregate, is performed. This analysis has shown that two elements seem to be important in the distribution of inter-centers distances: the inflection point and the spreading of all the distances. These two parameters characterize the isotropy of the aggregates shape. A good discrimination between the different convex hulls is reached with the two methods. However, they don't allow distinguishing the internal structure of the aggregates. For a better discrimination ofa ggregates, the authors are currently working on their geometrical characterization using more specific tools of point pattern analysis.

\section{References}

1. Jacquier, S., Gruy, F.: Anomalous diffraction approximation for light scattering cross section: Case of random clusters of non-absorbent spheres. J. Quant. Spectrosc. Radiat. Transfer, 109(5): 789-810 (2008)

2. Xu, Y.-1.: Electromagnetic scattering by an aggregate of spheres. Applied optics, 34(21): 4573-4588 (1995).

3. Lagarrigue, M. PhD thesis: Caractérisation optique et géométrique des agrégats multi-échelles. École nationale Supérieure des Mines de Saint-Étienne (in progress)

4. Mecke, K., Stoyan , D.: Finite packings and parametric density. Statistical Physics And Spatial Statistics. Springer, Klaus R. Mecke Dietrich Stoyan (Eds) : 332-845 (2000)

5. Santalo, L.: Integral geometry and geometric probability. Cambridge University Press (2004)

6. Ripley, B.D.: Modelling spatial patterns. J. Roy. Statistical Society, B 39: 172-212 (1977)

7. Gruy, F.: Light-scattering cross section as a function of pair distribution density J. Quant. Spectrosc. Radiat. Transfer, 110(3): 240-246 (2009) 